\title{
INFORMATION TECHNOLOGY AND AESTHETICS: PASSIVE AND ACTIVE DIMENSIONS
}

\author{
John D. Haynes \\ School of Information Technology \\ Faculty of Technology \\ Charles Darwin University \\ john.haynes@cdu.edu.au

\section{David B. Paradice} \\ Management Information Systems Department \\ College of Business \\ Florida State University \\ dparadice@cob.fsu.edu
}

\begin{abstract}
This paper is concerned with examining and recognizing aesthetics in an Information System (an organization incorporating both humans and information technology). Information Systems designers need to consider the aesthetics of their systems. To do this, they need some conception of what aesthetics is and is not. This paper constructs such a concept. IS designers must avoid thinking of a specific system component such as a graphical user interface as defining the aesthetic dimension of their work. Aesthetics emerge from the wholeness of things, not from specific parts or components. As such, aesthetics may naturally be considered in "systems", and we propose that an effective manner of thinking of aesthetics is in terms of "themes". We also argue that one must consider not only aesthetics that may be perceived by the senses (a passive dimension), but also aesthetics that may be conceived in the mind (an active dimension). We also consider the important differences between "definitions" (passive dimension) and "themes" (active dimension). Finally, the study of aesthetics in IS has greater implications than simply recognizing and appreciating beauty and art. The very human capacity for recognizing and appreciating beauty and art, the active aesthetic dimension, is also the same capacity for effective creativity and happiness. It follows that if an information system encouraged and provided the enabling circumstances for the human capacity of thematic recognition (as found in the human appreciation of art and beauty) it thereby also provides the grounds for effective creative and visionary organizational management.
\end{abstract}


Key words: aesthetics, information technology, information systems, active aesthetic dimension, thematic recognition, ethics, passive aesthetic dimension, perception, conception, C. West Churchman, Heidegger.

\section{INTRODUCTION}

To begin to consider the humanly appreciated notion of aesthetics in an information system is to already begin to consider more closely the human component of Information Systems. From this perspective we do not look literally at the Information System, but rather we look at it indirectly through the lens of Human Being. In so doing, we thus expose limitations in what it means to have a literal "systems view" closed to the ramifications of the genuine interaction between humans and information technology. Wilber (1998) describes - this kind of closed - systems theory as a "flatland holism" focused on objectifiable processes but lacking any connection to beauty, poetry, desire, love, moral wisdom, or artistic expression. Churchman (1971, 1982, 1994), a leading systems theorist, repeatedly made calls for the expansion of management science from literal, functional, closed, process considerations to the openness of the "thinking designer"; as he put it "to transform the success of the other functions into thinking” (Churchman, 1971, p 262). Churchman wanted management science to be infused with the 'living' vitality of human thinking. It follows that human thinking is far more "alive" when humans themselves are in a state of care and creativity. While Mitroff and Denton (1999) have identified the influential role of spirituality in the workplace in general, Armour (2002) identifies the "spirit" of projects as being the deciding factor in information systems success. Information systems are used to support decision-making processes used in resolving problems, and Mitroff and Linstone (1993) note that aesthetics play a fundamental role in the means we use to address problems. Kuhn (1962, p. 158) observes that sometimes "it is only personal and inarticulate aesthetic considerations" that can lead scientists to believe that a new proposal is on the right track. Dalal (2002) asks flatly, "Why do we not refer to the aesthetics of information systems as we do of works of art?”

Information Systems must be designed with aesthetics in mind for Dalal's request to be realized. This requires IS designers to have some conceptualization of aesthetics. Recognizing aesthetics is an important and crucial first step, but understanding aesthetics as it may apply to IS may be truly problematic. This paper provides a construction of the aesthetic concept that IS designers may employ. We feel that recognizing aesthetics is tantamount to recognizing what it is to be open for inspiration. Human inspiration and creative design become all the more effective when they are openly recognized to be important. Such self knowing enshrines and empowers the IS designer to become the "thinking designer". One crucial outcome from the self knowing of human creative design and genuine human inspiration is the realization that it is important in its own right.

It is open recognition that allowed ethics to emerge as a crucial consideration in the design of Information Systems. Ethics in information systems continues to receive attention, particularly as it relates to the proper handling and storage of information (Mason, 1986; Paradice, 1990; Banerjee, 1998; Davison, 2000). Ethics continues to be an important field precisely because it is of direct and recognized concern to the "thinking designer". The continued genuine interest in ethics and information systems does not arise because ethics is seen as an object in some systems process, but because it is recognized to be inherently part of what it is to be - and what it is to continue to be human. As other humans (clients, customers) recognize the ethical embrace in certain information systems they relate in a more holistic, genuine way to the information system and thus, especially in service industries, purchase more of its products. Ethics provides for the care of the human, and in a 
state of care humans are open to creativity, genuine action and also appreciation, among other purchase considerations, for a product produced in an ethical way.

While ethics appeals to the heart (the senses of care and preservation), aesthetics appeals to both the heart (in this case, the passions) and the mind (our sense of beauty). In the fields of philosophy and art, where aesthetics are normally considered, there is much debate over exactly what aesthetics encompasses and how it may be defined. The central conceptual construction that we pursue in this paper is to transform thinking about aesthetics in the context of information systems from a passive approach that relies on definitions and perceptions, into a vital "living" experiential open "thematic" and conceived active approach abundantly suited to C West Churchman’s “thinking designer”.

\section{PASSIVE APPROACH: DEFINITIONS: ACTIVE APPROACH: THEME}

Definitions are a crucial starting point for any endeavor, but they only provide a part or a glimpse of what they are describing. The risk is that they are somehow treated more than as a literal beginning and so are treated as an end in themselves. Because they are only a glimpse (or a part) of what they describe we refer to definitions as passive. To shift from the reliance on definitions of aesthetics (a passive approach) into a thematic approach (an active approach), thereby makes a crucial transformation, namely, to begin to act out (to re-cognize; to cognize again; to draw upon past experience). In an active approach, we take responsibility for thinking through past experiences of, in this case, aesthetic pleasures in relation to a current aesthetic circumstance. But when we compare a current experience with a prior one recognized as aesthetic, what we compare are themes. We do not literally compare each part of the past experience with each part of the new experience. We compare the overview, or theme, of each. This capacity for thematic comparison is important for this paper since thematic comparison is active and reflective. The more thematic comparison is achieved the more likely it is to become an effective capacity for active vision generally. The more an Information System reflects aesthetic dimensions, in what ever way it contextually does, the more opportunities there are for effective visionary output and management.

\section{AESTHETICS AND THE AESTHETIC DIMENSION OF LIFE}

Aesthetics is defined in a standard dictionary as "a branch of philosophy dealing with the nature of beauty, art, and taste and with the creation and appreciation of beauty." When we rely solely on definitions (a passive approach), we note an immediate limitation of subsequent discussions of aesthetics. These discussions run the risk of being unproductive simply because there are many different and seemingly unrelated definitions of beauty, art, and taste.

On the other hand, while not defining art, the philosopher Stephen Pepper does identify four themes (an active approach) of beauty, each corresponding to one of his four world hypotheses (Yanal, 2001).

- Mechanism, associated with Santayana, holds beauty to be the experience of immediate pleasure, and ugliness to be the experience of immediate displeasure.

- Contextualism, which Pepper associates with Dewey, holds beauty to be "voluntary vivid intuitions of quality." 
- Organicism, associated with Bosanquet, holds beauty to be an "integration of feeling”, and the degree of beauty varies with "the degree of integration and the amount of material integrated.” Finally,

- Formism, primarily associated with Aristotle, holds beauty to be the conformity of an object to the "norm" of the class of which it is a member. Pepper implies that one's worldview (or Weltanschauung) is integrally related to one's conception of beauty.

Like Pepper, those persons who are moved by beauty are moved in terms of themes which resonate personally with themselves. These themes are ways for them to connect with beauty. Such a connection cannot be made with a definition. A thematic approach more closely and directly connects to our hearts and our minds (we connect with a lived experience), whereas definitions appeal in an indirect way only to our minds through our senses. We perceive definitions, but we conceive when we relate with themes. Similarly we initially perceive works of art and, then in relation to their aesthetic capacities, we conceive them thematically.

We can see how the difference between a perception and a conception plays itself out in the following example. Consider works performed in a manner different from the original context for which they were intended. For example, consider a musician performing a composition created by another musician where the other musician's work is considered a work of art. Consider an extreme case, for example, the elementary school child who plays a composition and who faithfully executes each and every note of the composition. The same composition, also faithfully executed by a master musician, may be considered a work of art, yet the schoolchild's effort is not. The reason for this is that the child has not yet made the shift from perception to conception. The schoolchild is still working at a definitional stage, defining the notes and carefully reproducing them without fully comprehending the active power of the theme released by the notes. The master not only perceives the notes and the melody; but, conceives the full power of the theme of the music and thus is able to render the playing of the music with the full active vigor of that conception.

Similarly for designs in Information Systems, opportunities will be precluded if those designs limit themselves to perceptions of how the Information System works in terms of defining its operations or in comparison with other information systems operations. Perceptions are based on what already is the case, and in this sense they are present-oriented, or passive. But when design principles embrace the aesthetic dimension of an Information System, there is a focus towards what is not yet the case, and new questions emerge such as, "How can we provide the enabling circumstances for humans to be creative?" and "How can we provide the enabling circumstances for humans to be visionary?" These questions are questions of aesthetics. They are questions that relate to the human capacity to be creative: namely, a human's ability to conceive of thematic connections.

Haynes (1999) points out, themes cannot be perceived, but rather, they can only be comprehended through conception. We perceive colors and brush strokes and the outlines of, for example, a painting but we conceive of the theme that the painting portrays. A work of art portrays and offers its theme for inspection in much the same way that the concept of ethics offers its theme for consideration. In the case of the work of art, the beauty, taste, and artwork arise out of the whole state of the piece, the whole presentation, the whole accomplishment, which is itself thematic of its beauty, taste, artistry, etc. The beauty, taste, and art are not a part of the whole piece, but integral with it. We conceive these out of the whole in a thematic way. Similarly when ethics is genuinely embraced in an Information System, it is not the sets of rules of what it means to be ethical that portrays that ethic, but rather how ethics is conceived of by the IS organization, and how each member acts according to his or her own conception. We can perceive a mission statement on ethics, but that does not tell us if the IS is ethical. We can only come to know that by seeing how 
those rules are adopted. The adoption of those rules requires human interpretation. Interpretations are conceived rather than perceived.

Art is a product of culture and it is through art that a culture expresses its life (IEP, 2001). Similarly the culture inherent in an information system expresses something vital and living. If not, then there is an absence of culture in an information system in which case there is an absence of vitality. Thinking designers thrive in conditions of vitality. Like the theme in a work of art, a culture expresses itself as a theme and so it is not able to be strictly perceived (we perceive only its outward manifestation); but, rather it is more that the theme of the culture moves the thinking designer into action. Comprehending such movement entails conception, rather than perception.

\section{Passive Dimension (Perceptions); Active Dimension (Conceptions)}

On the passive side are the experiences we have when we take in the external beauties of the world. The passive aesthetic is defined in terms of what people see, hear, smell, touch, and taste. In other words what they perceive. On the active side are the experiences we have when we consider things that people do. The active aesthetic includes performances as a musician, athlete, or creative problem solver. In other words what we conceive.

A deeper conception of the aesthetic experience requires something well beyond the excitement of the senses, well beyond perception. That is to say, when the aesthetic experience is typically considered, the focus may be on how some art object looks or feels in its totality, or in the case of music: how it sounds in terms of the total performance. The perceptions of the senses and the conceptions of the totality of what is experienced cannot be divorced from prior experiences (IEP, 2001; van Gerwen, 1993). A particular work of art is first perceived as an object by the senses, and then is conceived to be beautiful in comparison with other objects - as a totality - that have been experienced. Indeed, to appreciate the "beauty" in an imaginative solution to a problem requires extending the concept of the aesthetic experience to include significant cognitive processes beyond simply touching, seeing, tasting, hearing, and smelling.

Two philosophers who gave aesthetics a great deal of attention were Immanuel Kant and John Dewey. According to Leddy (2001), Kant distinguished (in our terms) thematically between two types of beauty: free beauty and adherent beauty. Free beauties included birds, trees, music-not-setto-words, and even wallpaper. Adherent beauties, on the other hand, included people, horses, and buildings. The difference in the two categories is that adherent beauty depends on a notion of the purpose of the thing being considered. For some reason, Kant presupposes a notion of perfection for adherent beauties, and hence the beauty of them depends on their relationship to that ideal. Consequently, Kant can admire line art in one form, but dismisses the artwork of tattoos on humans because they interfere with the "perfection" of the human body.

Kant's moral philosophy emphasizes that pleasure cannot be the impetus for moral action. Kant's position is that pleasure varies from individual to individual, and so pleasure cannot be a basis for society-wide agreement on moral action (Donohoe, 2001). Agreement on the universal validity of a moral proposition could never be achieved if it were dependent on endorsement only when it provided personal pleasure. Kant therefore distinguishes between upper and lower faculties of desire. The lower faculty is driven by the pursuit of pleasure, the upper faculty by the pursuit of moral actions. Humans are partially sensuous and partially rational. Since all humans are driven by pursuits of pleasure, Kant introduces the idea of a moral "command" to do good, the categorical imperative. Tightly integrated in these notions is the idea that humans have the capacity of free will. It is the human ability to choose to do good that ultimately produces the pleasure associated with making moral choices. Consequently, pleasure is not the motivator for performing moral acts, but pleasure may be attained from doing them. 
Understanding Kant's concepts of moral behavior is critical to understanding his concept of the aesthetic experience and aesthetic judgment. According to Guyer (see Donohoe, 2001), Kant describes two separate acts of reflection in the making of an aesthetic judgment. The first act recognizes the pleasure attained. The second act uses the experience of the first act as evidence upon which to make an aesthetic judgment of the experience. It is in the second act of reflection that the original pleasure is categorized as either a satisfaction of a desire or the result of an aesthetic experience (Donohoe, 2001; van Gerwen, 1993).

Dewey also took a thematic approach and stressed the importance of recognizing the significance and integrity of all aspects of human experience (IEP, 2001). The roots of aesthetic experience lie, Dewey argued, in commonplace experience, in the consummatory experiences that are ubiquitous in the course of human life. Dewey felt that the creative work of artists was not unique: any intelligent use of materials or ideas to create imaginative solutions to problems could afford the same type of immediate satisfaction one feels when viewing a great work of art. Therefore, the aesthetic experience could be broadened to include dimensions of life not typically associated with the “creation and appreciation of beauty.”

Dewey also rejected the notion of the audience as a passive recipient of art (IEP, 2001). The one appreciating the art must assimilate the artist's work, once again in the context of that person's prior experiences. The appreciator must compare the current experience with prior experiences, integrate it into their personal experience, and perhaps discriminate among many possible types of prior experiences in order to assess the current feeling about the art being considered.

\section{WHY AN AESTHETIC DIMENSION CAN BE CONSIDERED IN INFORMATION}

\section{SYSTEMS}

Although it has hardly been examined in the IS literature, there is precedent for considering aesthetics in system design. Bansler et al. (2000) touched on the topic in a passing comment about web designers who perceived a need to improve the "look and feel" of an intranet environment. Markus and Mao (2001) note that developers may have a goal of producing an aesthetic design, but these researchers do not provide any indication at all of what the phrase "aesthetic design" means or implies. As an extension of these earlier considerations, one of the central conclusions of this paper is that a "look and feel" and an "aesthetic design" can only be genuinely (actively) considered in a thematic way. A genuine aesthetic design implies its own thematic recognition. A real capacity (a conception rather than a perception) for improving a "look and feel" in an IT environment comes from a thematic ability to connect who looks and feels with what is looked at and felt.

Wells (1996), in his examination of postmodernism and information technology, notes that Heidegger characterized the modern technology age as lacking in philosophical thought. We can take this to mean that the design of information systems are lacking in "thinking (philosophical) design". We suggest that consideration of a distinctly philosophical concept, aesthetics, can enhance the way information systems are "thought-fully" designed and consequently "thought-fully" discussed.

However, Heidegger (Heidegger, 2001) also provides an incentive for pursuing the goal of this paper. Consider the following quote from The Question Concerning Technology (section 35):

Because the essence of technology is nothing technological, essential reflection upon technology and decisive confrontation with it must happen in a realm that is, on the 
one hand, akin to the essence of technology and, on the other, fundamentally different from it. Such a realm is art.

We are not so much interested in the realm of art, but what constitutes essential reflection upon art, and to draw upon the capacity of such reflection, which is an aesthetic capacity, in the design of Information Systems. We have noted accordingly that such reflection comprehends a theme and it does so not by perception, but by conception. What is akin then to the essence of Information Systems is the theme of an Information System. We can conceive of a theme in general or a theme in particular. In terms of the particular design of an Information System, we direct our attention to aesthetic considerations, namely: what is thematic or could be thematic in a particular information system? Information technology is only part of an information system, and so in terms of an aesthetic thematic approach we must pay particular attention to the relationship between humans and information technology. It is crucial to see that relationship in a thematic way, that is, as information technology being used by humans. By providing for the enabling circumstances for the emergence of that kind of thematic viewing or aesthetic sense, in an information system, humans can draw inspiration from a relationship with information technology.

There are distinct dangers in not providing for the emergence of an aesthetic sense in an information system. This danger was noted by Wells who observes that Marcuse believes the nature of technology is to create a universal abstraction that alienates people from the world. Coyne (1995) observes how information technology can marginalize ethics, decontextualize the human experience, and provide a means for one person or group to dominate another. Introna (2002) argues that ethical considerations are becoming more difficult as information technology increasingly disassociates the consequences of decision makers' actions from the people affected by those decisions. Clearly, there are those who believe information technology is in need of some kind of "humanizing” experience.

Consideration of providing for the enabling circumstances for an aesthetic dimension in Information Systems can address these concerns. To summarize the above discussion and draw some implications:

- Definitions of beauty and art are not critical to our purpose. One need only recognize that there are thematic passive and active experiences that give pleasure, and that pleasure contributes to the aesthetic dimension of living.

Many have known the frustration (lack of pleasure) in being unable to acquire information in a timely manner, or in dealing with an information system that does not produce output in a form needed. When information is available as needed, when needed, and in the form needed, the information system has greater potential for providing pleasure on some aesthetic plane which in turn stimulates more effective action.

- Aesthetic experiences go beyond the five primary senses (perception) and include "performance" activities, including creative problem solving (conception). Indeed aesthetic experiences, as we have noted, are directly related to our human capacity for comprehending themes. If we no longer were confronted, or even limited in its expression, by beauty in any of its multifaceted forms, what would happen to our aesthetic sense? We have also noted that our aesthetic sense is directly connected to our capacity for effective long term vision.

Creativity is a hallmark of human capability. But creativity, or creative thinking, is distinct from aesthetics. All human creativity is an aesthetic experience drawn from the well-springs of the subtle capacity of conceiving and either that conception arises from an inner aesthetic mood or an outer aesthetic inspiration. In this sense we believe that conceiving (which contributes to the welfare of 
humans) is directly connected with the experience of aesthetics. Effective creativity requires excitement and a spirit of adventure where the imagination is, to a large extent, unrestricted. In simple terms if a workplace is drab - that is, unaesthetic - then creativity may be curtailed. If ideas are drab - and ideas also provide the basis for an aesthetic experience - then creativity will suffer accordingly. Creativity is a necessary prerequisite for good design and good design is directly connected with aesthetics: a good design is aesthetic. Thus effective creativity in particular produces aesthetics and the absorbed inspiration of aesthetics in general provides a ground for the possibility of effective creativity. In this way creativity is indirectly connected with aesthetics. Churchman observes that "design is one of the most fascinating" activities of man, (Churchman, 1971, p. 3) and that mankind's ability in creating art, science, religion, and politics is paramount. The design of information systems is no less a creative act. In fact, it may be that if we do not comprehend what it is to provide for effective human creativity in the design of information systems, we run the serious risk of limiting such precious human capacities in the future.

- According to Kant, if people are motivated by a will to perform moral acts, people are endowed with the necessary and sufficient prerequisite to cultivate the aesthetic dimension of life.

If Kant is correct, we must provide the enabling circumstances to allow for an aesthetic dimension to emerge and all the more so (for the above reasons) in an information system. Information systems workers are no less predisposed to moral action than any other professional group. In fact, studies have shown that information systems personnel conduct themselves in a manner consistent with concern for the well being of society (Udas et al., 1996; Paradice and Dejoie 1991). There is no reason to presuppose, therefore, that information systems workers are not capable of considering and ultimately enhancing the aesthetic dimension of information systems.

- Living a "good life" involves fulfillment on all dimensions of life.

People interact with information systems in many ways. Certainly, information systems pervade the workplace. People also utilize information systems when traveling (in the form of transportation schedules or the dynamic output of road signs), when relaxing at home (in the form of on-line entertainment guides), and when shopping (either on-line or to determine prices on items actually in stores or during the purchasing process) among other ways. Incorporating an aesthetic concern into information systems can have far-reaching implications for enhancing the ability to live a "good life". Why is living a "good life" thematic? Because what is of the good is thematic precisely because a person with good judgment conceives (comprehends the totality of it as theme) it to be so. You cannot have for example, a theme of evil. Evil is not capable of being thematic. It does not have sufficient quality to integrate itself into a whole; it is always fragmented. The beholding of beauty as an expression of living the "good life" on the other hand has the quality of being able to be unified and thus "exhibits or performs" itself thematically. If it is thematic it can be conceived by humans who have the wisdom and experience to do so and wisdom is fertile ground for "thinking designs”.

- $\quad$ The roots of the aesthetic experience are in everyday activities.

The ubiquitous nature of information systems certainly plays a role in everyday experiences. If an appreciation of an aesthetic dimension can be incorporated into information systems design, a widespread impact on the aesthetic dimension of people's lives can be realized.

- Aesthetics involves recognition of a pleasurable experience.

We need to carefully distinguish between fleeting pleasurable experiences and those pleasurable experiences that are based upon real past experiences. Aesthetic recognition entails drawing upon an experience and comparing that experience to the current experience. We have noted that this is an 
aesthetic capacity for comprehending themes. Such aesthetic experiences are pleasurable individually because our own experiences are involved. When we witness art at the level of perception and conception we are an audience to that art. Information systems, like most complex entities, may be perceived and conceived on many levels. In information systems, the audience may be users or information systems professionals. It follows that either user or professional, or both, will engage better if they are undergoing a pleasurable experience, and it will be all the more enduring if it is a pleasurable experience based upon conception. That is, the pleasurable experience was not just fleeting through perception, but connected - and thus conceived - to past pleasurable experiences.

As further confirmation of what we have previously elaborated as the need to distinguish between perception and conception, and the caution that it is conception that entails the aesthetic experience and not purely perception, Sibley (1959, p. 426) notes that something may be described in nonaesthetic terms "as fully as we please"; but, we are not thereby put in a position of having to admit that the thing is, in fact, aesthetic. In other words, we have a perception not yet confirmed by conception. We find evidence of this pervasively in advertising. For example, a sign gives us immediate pleasure by perceiving it, but it becomes fleeting if it is not grounded in previous lived experiences that genuinely gave us pleasure.

\section{PASSIVE AESTHETICS IN INFORMATION SYSTEMS - PERCEPTION}

The passive aesthetic of information systems design includes those aspects that are detected by our senses: seeing, touching, hearing, tasting, and smelling. The last two, tasting and smelling, are not normally excited by information system characteristics, and therefore will not be considered in depth here. However, a broader examination of how information systems technology is used in other domains could lead one to reasonably consider characteristics of taste and smell later.

Much has been studied that is related to what people sense when interacting with information systems. Beginning with the study of tabular data representation versus graphical data representation (DeSanctis, 1984; Jarvenpaa and DeSanctis 1988), the information systems literature has a long history in studying what may be more generally called media richness (Markus, 1994; ElShinnawy and Markus 1997). With regard to sight, the judicious choice of color combinations, use of fonts, use of effects such as blinking, use of transitions, use of special effects such as curled corners, and other visual cues has gained greater appreciation (Flanders and Willis, 1998) with the advent of multimedia support in systems.

The sense of touch is excited in the use of the keyboard, mice, track balls, pointer sticks in notebook keyboards, track pads, stylus, light pens, and similar input devices. In the design of information systems, most users have little control over the design of these hardware devices. Information systems professionals and academics may, however, need to give thought to the location of icons in an information system's interface since the location determines where one points. Information systems professionals and researchers need to pay attention to the concept of "focus" in interface design. An object in an interface "has focus" when it is ready to receive input. Users will likely find a design that moves focus from object to object in a natural progression to be more aesthetically pleasing than a design in which the focus moves in a seemingly random order.

With regard to hearing sensations, users typically experience sounds associated with events such as warnings, incoming mail, file deletion, and so forth. Where sounds are utilized in this manner, users need control over the sound used and its volume. Lack of such control, while not implicitly leading to a lack of aesthetic beauty in a system, could be an obstacle in attaining passive aesthetic quality. 
It is obvious that passive aesthetics related directly to users' and professionals' perceptions have undergone radical changes over the past several decades, precisely because passive aesthetics are a crucial design feature of current and future computer technology and systems. This trend will continue and all the more so with a better understanding of the relationship between human perceptions and human conceptions.

\section{ACTIVE AESTHETICS IN INFORMATION SYSTEMS - CONCEPTION}

Turning to the active aesthetic of information system design, focus must move to something more than the technology itself. Attention must turn to the information system user's objective in using the system. The need for an organization's information system strategy to be coordinated with its business strategy is accepted (Mata et al., 1995). Unlike the passive dimension, in which "measures" of aesthetically pleasing characteristics rely on the senses being excited, in the active aesthetic dimension the mark of aesthetic pleasure may occur when the system goes unnoticed. To put this point another way, much as a well-officiated sporting event is characterized by officials who essentially do not interfere with play (i.e., when the official does not interfere with the theme of competition), an information system that does not interfere with the user's goals is very likely to be viewed as pleasing by that user. Why? Because such a situation of minimal interference frees the user to use her imagination, and in so doing releases the user's conception, as an active aesthetic connection, with her past lived experiences.

For example, consider a small aspect of today's constantly changing business environment: telephone area code and postal code changes. While they are relatively stable, these codes do change. Many systems fill in these values automatically based on the entry of an address. Unfortunately, many shipping systems do not allow a manual override of these codes. In this case, the system has interfered with the goal of the user (efficient processing). The result can be that a customer is not notified in a timely manner about a change in an order status or a package may arrive late. These events may engender frustrations in the customer that ultimately become frustrations for the organization. The customer is then left with an inability to fulfill her goal. Thus, conception was blocked and a perception of frustration was created in its place.

Another aspect of the active aesthetic can be realized in the manner in which the information system organizes its information vis-à-vis the user's mental model. Wells and Fuerst (2000, 2001a, 2001b) have studied the role of the metaphor used in an information systems interface. They describe how metaphors are human derived models that apply tangible, concrete, recognizable objects (from a source domain) to abstract concepts and/or processes (in a target domain). Metaphor use is an effective means to improving a user's ability to understand an interface and use a system. Clearly, the use of metaphor is an activation of conception in the user (Haynes and Mahfouz, 2001).

Wells and Fuerst discuss the advantages of, for example, the use of an airplane schematic in seat selection while making on-line ticket reservations or the ability to place furniture in a virtual room while furnishing an office or home. However, problems can arise when an incorrect metaphorical model is used, when the metaphor is unrealistic, or when a mixed metaphor occurs. When the metaphor is appropriate, the user's learning curve is flatter and productivity, information retention, satisfaction, and accuracy increase. Wells and Fuerst claim that deriving the interface from the appropriate domain is attractive because it couples the user's view of the domain with the designer's view, thereby reducing the likelihood of employing an incorrect metaphor. The proper metaphor leads to an aesthetically pleasant experience that has elements of both the passive and active dimensions. The passive aspect is inherent in the perceived connection a user will likely make between the system's intent and its interface. The active aspect comes into play when the user is 
better able to execute the tasks at hand, because conception now comes into effect, due to the better "fit" of the system and the task domain.

A final way to consider the active aesthetic in information systems occurs in the design of knowledge-based systems. Of interest here is the more general class of systems that are inquiring systems (Churchman, 1971; Courtney, 2001; Courtney et al., 1997 and 1998; Haynes, 2001; Kienholz, 1999; Richardson et al., 2001). According to Churchman (1971, p. 8), "Inquiry is an activity which produces knowledge." At the heart of any inquiring system should be an epistemology, a theory of knowledge. The essence of this theory is how people answer the question of how certain things are known to be true and other things to be false (Churchman, 2001). Clearly here we have a high level of conception tried and tested over, in some cases, lifetimes of experience.

Churchman outlines five archetypal inquiring system designs based on his understanding of the works of five philosophers (Leibnitz, Locke, Kant, Hegel, and Singer). The designs are not exact accounts of how each philosopher conceived the theory of knowledge. Rather, the designs are derived from a reconstruction of each philosopher's ideas in the language of the design of an inquiring system (Churchman, 1971, p. 18). In each case, in our terms, conception is the key.

Consider the use of conception in the following example for a Leibnizian-type enquirer. In evaluating an information system the evaluator will tend to work through assumptions and proposed facts, examining each for validity vis-à-vis her Weltanschauung. As each assumption or proposal is validated to our user, she will build an ever-enlarging fact net designed to "prove" some point. Suppose the system, on the other hand, is based on a guarantor of consensus. A new "fact" to this system can only be considered in terms of prior occurrences of the same category of facts for which some consensus has been achieved. Thus, on the first occurrence of a new fact, the system can only make note of its occurrence. There is no consensus (yet) regarding the fact. So, while the user may deduce the truth of the new fact using Leibnizian-type inquiry, her system fails to produce similar results. When the underlying epistemology of an inquiring system is not congruent with the user's epistemology, the inquiring system will be considered less aesthetically pleasing than when the user and the system share similar concepts of knowledge production. In other words, when the conception of the user does not evoke from prior experience a match to the current experience the consequences are not pleasing; when there is a match there is an aesthetically pleasing outcome.

\section{INFORMATION SYSTEMS DESIGN: PASSIVE DIMENSIONS AND ACTIVE DIMENSIONS}

We have examined the passive and active dimensions of an information system in relation to aesthetics, respectively, in terms of:

1. Definitions and themes.

2. Perceptions and conceptions.

3. Passive aesthetics outputs based on perceptions, and active aesthetics outputs based on conceptions.

Our focus throughout the paper has been on examining and recognizing two distinct aspects of aesthetics in relation to humans in the context of an information system. These two primary differences are in human perceiving and human conceiving, and both allow us to see the crucial importance of aesthetics for an information system. Effective visionary computer technology design 
focuses on the realm of human aesthetic perception, but first the designer must have conceived this to be the case. That is, the designer must have conceived that users are personally pleased to be bodily involved at the level of their senses. Effective visionary management design, which is at the same time ethical, draws its inspiration from the realm of aesthetic conception. In both cases conception is the key, and aesthetics is a crucial element. Moreover, the effective use of conception requires an environment that nurtures that conception.

Vital, active designs are "thinking” designs, but they cannot be thinking designs without aesthetics and without conception. With a sense of aesthetics and a capacity to draw upon conception, and with both in full measure, the designer becomes an artist. When the designer becomes an artist, that artistry transforms technology and gives it an appropriate place in the context of that information technology's association with humans.

\section{GUIDELINES FOR CONSIDERING THE AESTHETIC DIMENSION}

In closing, there are several guiding thoughts that may be put forward with regard to considering an aesthetic dimension of information systems.

\section{The aesthetic dimension is not characterized as "plug and play".}

In other words, there is no aesthetics module that can be added to a system to make it aesthetically pleasing. There is no component that can be inserted into a system to address the aesthetic dimension. Similarly, the lack of a particular component or characteristic does not imply a system lacks aesthetic beauty. The aesthetic dimension reflects itself in the holistic concept of human conception.

\section{The aesthetic dimension is not described by graphical user interfaces.}

Perhaps this is a corollary to the preceding point. When it was novel, the (generic) graphical user interface may have contributed to a system's aesthetic quality. A desk-top metaphor may be completely inadequate for a task that involves surgery, for example, or operating a complex machine.

\section{Aesthetics occurs when the system elevates the work to a new level of effective vision.}

Singer (1923, p.54) argued that "Beauty is that quality of a work of art which makes its tragic turning a moment inspired by an heroic mood of new creating." His argument is that only when one is struggling in some situation, and then confronts something that elevates that person beyond her struggle, does one recognize aesthetic quality. He continues (1923, p. 54), "The value of beauty to life is its inspiration to a soul in travail...". All vision is born of struggle, and it is individually the struggle to reconcile one's past experiences of creating moments of joy with one's current ones.

\section{Aesthetics may occur on different levels or from different perspectives.}

All complex systems are characterized by multiple stakeholders with often differing perspectives. Information systems are no different. There is a technical perspective that is reflected in the design and construction of the system. There is a personal perspective that is reflected in the manner in which a user interacts with the system. There is a domain perspective reflected in the manner the system accomplishes its purpose in a specific task domain. The algorithm employed could be aesthetically pleasing while the system itself (as perceived by the user) may not. 
According to philosophers, humans seek happiness on several dimensions, one being the aesthetic dimension. Aesthetics evolved as the "philosophy of art", but it actually extends beyond the art world. In fact, when people experience aesthetic pleasure in the routine of their lives, they truly begin to "live the good life." Churchman (1971, p. 199) notes: "The contented life is the complete life, made up of all those aspects of life that make it meaningful." One "lives the good life" when one is successful in finding happiness on all dimensions of life, and when one creates opportunities for others to do likewise. Since information systems permeate modern life, developing the concept of an aesthetic dimension of information systems can have a great impact on the development of the aesthetic dimension of life in general.

\section{CONCLUSION}

We have demonstrated in this paper the construction of the concept of aesthetics in terms of two dimensions. The second dimension, the active, goes well beyond our perceptions (the passive dimension) in the sensations we feel when one of the five senses is stimulated. Aesthetic pleasure can also be experienced in various areas of performance, including creative problem solving as well as the everyday function of people's lives where all of these areas draw on our lived experiences; thus, enriching aesthetically the conception of our lives and by effect the lives of others. Therefore, two areas in which the aesthetic dimension of information systems exist have been identified and explored. One is the passive aesthetic dimension, which focuses on the parts of the system seen, touched, and heard (namely perceptions) and we also articulated this dimension in terms of "definitions". The second is the active aesthetic dimension, which focuses on the performance of the system and, in the case of inquiring systems, on the system's underlying epistemology and we also explicated performance in terms of conception and further, its connection to creativity. We have also explored the view that the active aesthetic dimension can apply to the art of management, insofar as a comprehension of aesthetic considerations, together with ethical considerations, can provide a basis for visionary management.

Recognizing the need to attain fulfillment through happiness, and the role of the aesthetic dimension in doing so, can be a guiding force in the evaluation of information systems. Information system designers must incorporate ways for users to modify and control system features that fall in the passive aesthetic dimension, so that users may customize these features in ways that please them. Information system designers must be sensitive to the active aesthetic dimension, recognizing that the way a system functions must be consistent with both human and business information processing in order to create an opportunity for a pleasurable aesthetic experience for users. Designers, however, must not fall prey to believing that aesthetic quality can be added to a system by simply programming in a particular feature. "Success" on the aesthetic dimension requires that the information system elevate a user to a new and better environment and way of life. The more information systems professionals are able to consider this goal, and it can only be considered effectively thematically, that is actively, the more likely their systems will contribute to a better life for everyone. Such considerations arise from a recognition of the importance of aesthetics, which itself arises from out of a deliberate organizational provision for the enabling circumstances of care and creativity; a deliberate provision for providing human organizational members with the capacity for thematic recognition. In our view, it is a necessary provision, because the relationship between a human being and information technology, in an organization, is always imminently aesthetic and therefore in that imminence, there is always the potential for evoking effective creativity. 


\section{REFERENCES}

Armour, P. (2002) “The Spiritual Life of Projects”, Communications of the ACM, Vol 45 No 1, pp11-14.

Banerjee, D. (1998) “Modeling IT Ethics: A Study of Situational Ethics”, MIS Quarterly, Vol 22 No 1, pp 31-60.

Bansler, J.P., Damsgaard, J., Scheepers, R. Havn, E. and Thommesen, J. (2000) “Corporate Intranet Implementation: Managing Emergent Technologies and Organizational Practices”, Journal of the Association of Information Systems, Vol. 1, Paper 10.

Churchman, C. (1971) West. The Design of Inquiring Systems, Basic Books.

Churchman, C.W. (1982) Thought and Wisdom Intersystems Publications, Seaside, CA.

Churchman, W. (1994) "Management Science: Science of Managing and Managing of Science”, Interfaces, Vol 24 No 4, pp 99-110.

Churchman, C. West. (2001) “A Philosophy for Complexity, from Managing Complexity”, http://www.haas.berkley.edu/ gem/essays/complex.html.

Courtney, J. F. (2001) "Decision Making and Knowledge Management in Inquiring Organizations: Toward a New Decision-Making Paradigm for DSS,” Decision Support Systems, Vol 31 No 1 , pp 17-38.

Courtney, J.F., Croasdell D.T. and Paradice, D. B., (1997) “Lockean Inquiring Organizations: Guiding Principles and Design Guidelines for Learning Organizations,” Proceedings of the Americas Conference on Information Systems, Indianapolis, Indiana, August 15-17, pp 323325.

Courtney, J.F., Croasdell D.T. and Paradice, D. B., (1998) “Inquiring Organizations,” Australian Journal of Information Systems, Vol 6 No 1, September, pp 3-15, and Foundations of Information Systems: Towards a Philosophy of Information Technology,http://www.cba.uh.edu/ parks/fis/fisart.htm.

Coyne, R. (1995) Designing Information Technology in the Postmodern Age. Cambridge, MA: The MIT Press.

Dalal, N. (2002) “An Integral Perspective of Information Systems”, Proceedings of the Eighth Americas Conference on Information Systems, Dallas, pp 1710-1713.

Danto, Arthur C.(2001) “Art, Philosophy, and the Philosophy of Art”, http://www.csulb.edu/ jvancamp/361/361_r1.html.

Davison, R.M. (2000) "Professional Ethics in Information Systems: A Personal Perspective”, Communications of the Association of Information Systems, 3(8), pp 1-34.

DeSanctis, G. (1984) “Computer Graphics as Decision Aids, Directions for Research”, Decision Sciences, Vol 15 No 4, pp 463-487.

Donohoe, B., (2001) “An Examination of Moral Action and Aesthetic Judgment in Kant’s Critical Philosophy”, http://www.janushead.org/JHspg99/donohoe.cfm.

El-Shinnawy, M. and Markus, M. L. (1997) “The poverty of media richness theory: Explaining people's choice of electronic mail vs. voice mail”, International Journal of HumanComputer Studies, Vol 6 No 4, pp 443-467. 
Flanders, V. And Willis, M. (1998) Web Pages That Suck: Learn Good Design by Looking at Bad Design.

Haynes, John D. (1999) "Practical and Tacit Knowing As a Foundation of Information Systems," Australian Journal of Information Systems, May, No 6, pp 57-64.

Haynes, John D. (2001) "Churchman’s Hegelian Inquiring System and Perspectival Thinking," Information Systems Frontiers, Vol 3 No 1, pp 29 - 39.

Haynes, John. D. and Mahfouz, A. (2001) "Objects That Create Their Own Context: The Inclusion of Conception in the Mental Model of the User in Interface Design," Proceedings of the Computer Human Interaction Conference, UCOL \& Massey University, Palmerston North, New Zealand, pp 39 - 43.

Heidegger, M. (2001) The Question Concerning Technology, http://www.centenary.edu/ balexand/cyberculture/question1.html.

IEP (2001) The Internet Encyclopedia of Philosophy, http://www.utm.edu/research/iep/.

Introna, L.D. (2002) “The (im)possibility of ethics in the information age,” Information and Organization, Vol 12, pp 71-84.

Jarvenpaa, S. and DeSanctis, G. (1988) "Standard Scaling in Decision Graphs: An Empirical Investigation”, IEEE Transactions on Systems, Man, and Cybernetics, Vol 18 No 5, pp 824831.

Kienholz, Alice (1999) "Systems ReThinking: An Inquiring Systems Approach to the Art and Practice of the Learning Organization,” http://www.cba.uh.edu/ parks/fis/fisart.htm.

Kuhn, Thomas. (1962) The Structure of Scientific Revolutions. Chicago: University of Chicago Press.

Leddy, T. (2001) “Kant’s Aesthetics: Tattoos, Architecture, and Gender-Bending”, http://aestheticsonline.org/ideas/leddy.html.

Markus, M. L. (1994) “Electronic Mail as the Medium of Managerial Choice”, Organization Science, Vol 5 No 4, pp 502-527.

Markus, M.L. and Mao, J.-Y. (2004) "Participation in Development and Implementation - Updating an Old, Tired Concept for Today's IS Contexts”, Journal of the Association of Information Systems, Vol 5 No 11-12, pp 514-544.

Mason, R.O.(1986) “Four Ethical Issues of the Information Age”, MIS Quarterly, Vol 10 No 1, pp 5-12.

Mata, F., Fuerst, W.L., and Barney, J. (1995) "Information Technology and Sustained Competitive Advantage: A Resource-Based Analysis,” MIS Quarterly, Vol 19 No 4, December, pp 487506.

Mitroff, I. and Denton, E. (1999) “A Study of Spirituality in the Workplace”, Sloan Management Review, Summer, 83-92.

Mitroff, I. and Linstone, H.A. (1993) The Unbounded Mind: Breaking the Chains of Traditional Business Thinking, New York: Oxford University Press.

Paradice, D.B. (1990) "Ethical Attitudes of Entry-Level MIS Personnel." Information and Management, 18, pp 143-151. 
Paradice, D.B. and Dejoie, R.M. (1991) "The Ethical Decision-Making Processes of Information Systems Workers.” Journal of Business Ethics, 10, pp 1-21.

Richardson, S. M., Courtney, J.F., and Paradice, D.B., (2001) “An Assessment of the Singerian Approach to Organizational Learning: Cases from Academia and the Utility Industry," Special Issue of Information Systems Frontiers on Philosophical Reasoning in Information Systems Research, Vol 3 No 1, pp 49-62.

Sibley, F. (1959) “Aesthetic Concepts”, The Philosophical Review, 68(4), October, pp 421-450.

Singer, E.A. (1923) On the Contented Life. New York: Holt \& Company.

Udas, K., Fuerst, W.L., and Paradice, D.B. (1996) “An Investigation of Ethical Perceptions of Public Sector MIS Professionals.” Journal of Business Ethics, 15(7), July, pp 721-734.

Van Gerwen, Rob. (1993) “Kant’s Regulative Principle of Aesthetic Excellence: The Ideal Aesthetic Experience”, presented at the Annual Conference of the British Society of Aesthetics, http://eserver.org/philosophy/aesthetic-excellence.txt, London, September.

Wells, John D. (1996) "Postmodernism and Information Technology: Philosophical Perspectives and Pragmatic Implications,” Proceedings of Second Americas Conference on Information Systems, Phoenix, pp 602-4.

Wells, John D. and Fuerst, William L. (2000) "Domain-Oriented Interface Metaphors: Designing Web Interfaces for Effective Customer Interaction,” Proceedings of the Thirty-Third Hawaii International Conference on System Sciences.

Wells, John D. and Fuerst, William L. (2001a) “Domain-Oriented Interface Metaphors: An Alternative Approach to Customer Interface Design,” Washington State University, working paper.

Wells, John D. and Fuerst, William L. (2001b)“Deriving Interface Metaphors from the Business Domain: An Empirical Investigation,” Washington State University, working paper.

Wilber, K. (1998) The Marriage of Sense and Soul, New York: Random House.

Yanal, Robert J., (2001) “Pepper on Definitions and Aesthetics”, http://www.sunyit.edu/ harrell/Pepper/pep_yanal.htm. 